\title{
Regularity of a class of degenerate elliptic equations
}

\author{
Qiaozhen Song, Ying Lu, JiAnzhong Shen And LiHe WANG
}

\begin{abstract}
In the present paper we establish the $W^{1, p}$ type estimates for the weak solutions of a class of degenerate elliptic equations. The optimal estimates are obtained by introducing the intrinsic metric that is associated with the geometry of the operator and then using the compactness method.
\end{abstract}

Mathematics Subject Classification (2010): 35J70 (primary); 35H20 (secondary).

\section{Introduction}

In this paper we consider the following degenerate elliptic equation:

$$
\mathcal{L} u:=u_{x x}+|x|^{2 \sigma} u_{y y}=f_{x}+|x|^{\sigma} g_{y} \quad(x, y) \in \Omega,
$$

where $\sigma$ is an arbitrary positive real number and $\Omega$ is a bounded domain in $\mathbb{R}^{2}$ with $(0,0) \in \Omega$ or $(0,0) \in \partial \Omega$.

When $\sigma$ is a positive integer, $\mathcal{L}$ belongs to a class of sum-of-squares operators, i.e.,

$$
\mathcal{L}=X_{1}^{2}+X_{2}^{2},
$$

by choosing $X_{1}=\partial_{x}, X_{2}=x^{\sigma} \partial_{y}$. The vector fields $X_{1}, X_{2}$ are smooth and satisfy Hörmander's condition that is the vector fields together with their commutators of some finite order span the tangent space at any point. In [7], Hörmander proved the following subelliptic estimate

$$
\|u\|_{H^{\varepsilon}\left(B_{\frac{1}{2}}\right)} \leq C\left(\|\mathcal{L} u\|_{L^{2}\left(B_{1}\right)}+\|u\|_{L^{2}\left(B_{1}\right)}\right),
$$

and as a consequence $\mathcal{L}$ is hypoelliptic. A few years later, Kohn studied the same problem by using some basic properties of pseudo-differential operators (see [9]). Furthermore, by studying the properties of the nilpotent group which is suitable to the analysis of the operator, Rothschild and Stein gave the optimal regularity estimates of $\mathcal{L}$ in various Sobolev spaces and Lipschitz spaces (see [12]). 
As $\sigma$ is an arbitrary positive number, all of the above methods do not apply since the vector fields $\partial x,|x|^{\sigma} \partial y$, are only Hölder continuous and do not satisfy the Hörmander's condition. In this setting, there are some papers studied the Hölder regularity of equation (1.1) in the last few years. For instance, Franchi, Lanconelli and Serapioni proved the Sobolev-Poincaré inequality, the Harnack type inequality and the $C^{\alpha}$ estimates of the weak solutions(see [3-5]). Their results are obtained by adjusting the classical Morse iteration techniques to the geometry of the homogeneous space that is related to the intrinsic metric(or currently called Carnot-Carathéodory metric) associated with the vector fields $\partial x,|x|^{\sigma} \partial y$. Moreover, in [14], one of the authors, Wang, gave an optimal Hölder estimate which is $C_{*}^{2+\alpha}$ regularity for all $\alpha<1$.

In this paper we will consider optimal $W^{1, p}$ type estimates of equation (1.1) for arbitrary $\sigma>0$. We must point out that the standard procedure such as RothschildStein's method does not work for our situation since the vector fields are not even smooth in $\mathbb{R}^{2}$ when $\sigma$ is not an integer. Our results are the following

Theorem 1.1. Let $1<p<\infty$ and $(0,0) \in \Omega$. If $u$ is a weak solution of equation (1.1) in $\Omega$, then $u$ satisfies a uniform estimate in any subset $\Omega^{\prime} \subset \subset \Omega$

$$
\int_{\Omega^{\prime}}\left(\left|u_{x}\right|^{p}+\left.\left.|| x\right|^{\sigma} u_{y}\right|^{p}\right) d x d y \leq C \int_{\Omega}\left(|u|^{p}+|f|^{p}+|g|^{p}\right) d x d y,
$$

where $C$ depending only on $\sigma, p, \Omega^{\prime}$ and $\Omega$.

Here we say $u$ is a weak solution of the equation (1.1) in $\Omega$ if

$$
\int_{\Omega}\left(u_{x} \varphi_{x}+|x|^{2 \sigma} u_{y} \varphi_{y}\right) d x d y=\int_{\Omega}\left(f \varphi_{x}+|x|^{\sigma} g \varphi_{y}\right) d x d y,
$$

for every $\varphi \in H_{0, *}^{1}(\Omega)$ (for the explicitly definition of the space see Section 3).

If the degenerate line is a part of the boundary $\Omega$, we give the following theorem.

Theorem 1.2. Let $1<p<\infty$ and $(0,0) \in \partial \Omega$. If $u$ is a weak solution of equation (1.1) in $\Omega \subset \mathbb{R}_{x}^{+} \times \mathbb{R}_{y}$ with $u=0$ on $T=\partial \Omega \cap\{x=0\}$ then, for any domain $\Omega^{\prime} \subset \subset \Omega \cup T$, we have

$$
\int_{\Omega^{\prime}}\left(\left|u_{x}\right|^{p}+\left.\left.|| x\right|^{\sigma} u_{y}\right|^{p}\right) d x d y \leq C \int_{\Omega}\left(|u|^{p}+|f|^{p}+|g|^{p}\right) d x d y,
$$

where $C$ depending only on $\sigma, p, \Omega^{\prime}$ and $\Omega$.

The key method of our approach is the compactness method and the idea follows what was introduced by Wang in [15]. In that paper, Wang gave a new proof of the classical Calderón-Zygmund estimate for the Laplace operator. After that, this geometric approach has been used to prove the regularity of the more general elliptic equations(see [1]). In this paper we show that it is also valid for the degenerate elliptic equations. 
Although the methods to prove the regularity for the elliptic equation and the degenerate one are similar, there are some difficulties of the proof since the operator $\mathcal{L}$ is lacking of ellipticity when $|x|^{\sigma}$ vanishes. As we know the Euclidean distance related to the Laplace operator, for the degenerate elliptic operator we need a suitable metric that is relate to the vector fields $\partial x,|x|^{\sigma} \partial y$. Hence, the balls, the maximum functions and so on, should also be adapted for our operator. For the elliptic equation the points are equal from the geometry point of view, so it is enough to give the estimates on the unit ball. For the degenerate equation, we must consider the estimates on each ball and then match the estimates on the balls centered at any point to obtain an uniform estimate.

We remark that our method can be applied to study the general degenerate elliptic equations in higher dimension.

The organization of the paper will be as follows: In Section 2 we give the definitions and the properties of the intrinsic metric and the analytic tools, such as the maximal principle and the covering lemma. In Section 3 we introduce the Sobolev type spaces associated with the operator. In addition, we give a new simple proof of the Sobolev-Poincaré type inequality by using the classical one. In Section 4 we give the interior $W^{1, p}$ type estimates of the weak solutions of equation (1.1) and the special boundary value problems.

\section{Preliminaries}

\subsection{The metric and the balls}

Now we introduce the metric related with the operator $\mathcal{L}$ that is given by:

$$
d s^{2}=d x^{2}+|x|^{-2 \sigma} d y^{2} .
$$

For any two points $P_{1}$ and $P_{2}$, denote this intrinsic metric as $\tilde{d}\left(P_{1}, P_{2}\right)$.

Since there is a natural scaling of the operator $\mathcal{L}$, that is,

$$
\mathcal{L}\left(u\left(r x, r^{1+\sigma} y\right)\right)=r^{2}(\mathcal{L} u)\left(r x, r^{1+\sigma} y\right),
$$

we expect there are some scaling properties for the metric and the balls. So for our convenience, define the following equivalent metric explicitly (see [14]),

$$
d\left(P_{1}, P_{2}\right)=\left|x_{1}-x_{2}\right|+\frac{\left|y_{1}-y_{2}\right|}{\left|x_{1}\right|^{\sigma}+\left|x_{2}\right|^{\sigma}+\left|y_{1}-y_{2}\right|^{\frac{\sigma}{1+\sigma}}},
$$

for any two points $P_{1}=\left(x_{1}, y_{1}\right)$ and $P_{2}=\left(x_{2}, y_{2}\right)$. The two defined metrics $\tilde{d}$ and $d$ have the following relationship,

$$
\alpha_{1} \tilde{d}\left(P_{1}, P_{2}\right) \leq d\left(P_{1}, P_{2}\right) \leq \alpha_{2} \tilde{d}\left(P_{1}, P_{2}\right),
$$

where $\alpha_{1}$ and $\alpha_{2}$ are positive constants depending only on $\sigma$. 
From now on and towards the end of this paper we fix

$$
\gamma=\frac{\alpha_{2}}{\alpha_{1}}
$$

then $\gamma \geq 1$ and depends only on $\sigma$. It is easily seen that $d\left(P_{1}, P_{2}\right)$ is a quasi-metric and satisfies

$$
d\left(P_{1}, P_{2}\right) \leq \gamma\left(d\left(P_{1}, P_{3}\right)+d\left(P_{3}, P_{2}\right)\right)
$$

Let $P=(x, y)$. Using the scaling property, we denote

$$
r P=\left(r x, r^{1+\sigma} y\right)
$$

then

$$
d\left(r P_{1}, r P_{2}\right)=r d\left(P_{1}, P_{2}\right)
$$

Define the ball with the center point $P$ as

$$
B(P, r)=\{X: d(X, P)<r\}
$$

and the intrinsic ball as

$$
\mathcal{B}(P, r)=\{X: \tilde{d}(X, P)<r\}
$$

We denote $B(0, r), \mathcal{B}(0, r)$ by $B_{r}, \mathcal{B}_{r}$, for simplicity. For any measurable set $\Omega$, $|\Omega|$ is its measure.

These balls have the following properties:

(a) For the intrinsic and explicitly defined balls, by (2.1), it is easily seen that

$$
B\left(P, \alpha_{1} r\right) \subset \mathcal{B}(P, r) \subset B\left(P, \alpha_{2} r\right)
$$

(b) The measures of the balls with the same center are controllable, that is,

$$
|B(P, r)| \leq|B(P, R)| \leq\left(\frac{R}{r}\right)^{2+\sigma}|B(P, r)|, \text { for } R \geq r>0
$$

We remark that the regularity results can be obtained by using the intrinsic metric. Since the explicitly defined balls are equivalent to the intrinsic ones, for our convenience, in many cases we use them to substitute the intrinsic ones, and then the equivalent results can be obtained. That can been seen in the later sections. 


\subsection{The maximal functions and the covering lemmas}

Since the regularity of the solution depends on the geometry of operator, the main analytic tools of the classical ones, such as the maximal function and the covering lemma, can not be used, so they must be adapted for the geometry of degenerate operator. Define the maximal function of a locally integrable function $f$ as

$$
\mathcal{M} f(X)=\sup _{r>0} \frac{1}{|B(X, r)|} \int_{B(X, r)}|f(x, y)| d x d y .
$$

Here the ball $B(X, r)$ can be replaced by the intrinsic one. For an integrable function $f$ defined in $\Omega$, define the local maximal function as

$$
\mathcal{M}_{\Omega} f(X)=\mathcal{M}\left(f \chi_{\Omega}\right)(X) .
$$

Remark. If a domain $\Omega$ is very close to $|x|=1$ and the diameter of $\Omega$ is very small, then in $\Omega$, the metric related with $\mathcal{L}$ is

$$
d s^{2}=d x^{2}+|x|^{-2 \sigma} d y^{2} \sim d x^{2}+d y^{2} .
$$

That means for $X \in \Omega$ and $r$ small, the intrinsic ball $\mathcal{B}(X, r)$ and hence $B(X, r)$, are equivalent to the Euclidean ball. If $r$ is large, then the measure of the set $\{f(x, y)=$ $0\}$ is large, so the average of $|f(x, y)|$ on $B(X, r)$ has little effect on the value of $\mathcal{M}_{\Omega} f(X)$. Thus the local maximal function defined here is equivalent to the classical one. Let $u(x, y)$ be defined in $B\left(X_{0}, r\right)$. Notice the scaling property of the operator $\mathcal{L}$ and let

$$
\tilde{u}(x, y)=u\left(\left|x_{0}\right| x,\left|x_{0}\right|^{1+\sigma} y\right),
$$

then $\tilde{u}$ is defined in $B\left(\frac{X_{0}}{\left|x_{0}\right|}, \frac{r}{\left|x_{0}\right|}\right)$. If the distance of the ball $B\left(X_{0}, r\right)$ to the degenerate line $x=0$ is very larger than $r$, then the ball $B\left(\frac{X_{0}}{\left|x_{0}\right|}, \frac{r}{\left|x_{0}\right|}\right)$ is close to $|x|=1$ and the diameter is small. For $\tilde{u}$, the local maximal function is equivalent to the classical one. Thus, if $\tilde{u}$ has some properties then, scaling back, $u$ has the similar properties. This is very useful and by using this property we can match the estimates at different points.

Applying the usual methods as in [13], we have the following Vitali covering lemma and the results of the maximal functions.

Lemma 2.1. Let $E$ be a measurable set which is covered by a class of balls $\mathcal{B}\left(X, r_{X}\right)$. Suppose the radius of $\mathcal{B}\left(X, r_{X}\right)$ are bounded, then there exists a disjoint sequence, $\mathcal{B}\left(X_{1}, r_{X_{1}}\right), \mathcal{B}\left(X_{2}, r_{X_{2}}\right), \ldots$, such that $E \subset \cup_{i} \mathcal{B}\left(X_{i}, 5 r_{X_{i}}\right)$.

By Lemma 2.1 and $B\left(P, \alpha_{1} r\right) \subset \mathcal{B}(P, r) \subset B\left(P, \alpha_{2} r\right)$, we immediately have the following equivalent result for the explicitly defined balls.

Lemma 2.2. Let $E$ be a measurable set which is covered by a class of balls $B\left(X, r_{X}\right)$. Suppose the radius of $B\left(X, r_{X}\right)$ are bounded, then there exists a disjoint sequence, $B\left(X_{1}, r_{X_{1}}\right), B\left(X_{2}, r_{X_{2}}\right), \ldots$, such that $E \subset \cup_{i} B\left(X_{i}, 5 \gamma r_{X_{i}}\right)$.

We need the following lemma which is usually called a modified Vitali Lemma. 
Lemma 2.3. Let $C \subset D \subset \mathcal{B}_{1}$ be two measurable sets contained in $\mathcal{B}_{1}$. Assume there exists an $\epsilon$ such that

1) $|C|<\epsilon\left|\mathcal{B}_{1}\right|$;

2) For every $X \in C$ and $r<2$, if $|C \cap \mathcal{B}(X, r)| \geq \epsilon|\mathcal{B}(X, r)|$, then $\mathcal{B}(X, r) \cap \mathcal{B}_{1} \subset D$.

Then

$$
|C| \leq\left(7 \gamma^{3}\right)^{2+\sigma} \epsilon|D| .
$$

Proof. Since $|C|<\epsilon\left|\mathcal{B}_{1}\right|$, for almost every $X \in C$, there is an $r_{X}<2$ such that $\left|C \cap \mathcal{B}\left(X, r_{X}\right)\right|=\epsilon\left|\mathcal{B}\left(X, r_{X}\right)\right|$, and $|C \cap \mathcal{B}(X, r)|<\epsilon|\mathcal{B}(X, r)|$, for $r>r_{X}$. By Lemma 2.1, there exists a disjoint sequence, $\mathcal{B}\left(X_{1}, r_{X_{1}}\right), \mathcal{B}\left(X_{2}, r_{X_{2}}\right), \ldots$, such that $(C \backslash A) \subset \cup_{i} \mathcal{B}\left(X_{i}, 5 r_{X_{i}}\right)$, where $A$ is a set of measure zero. So

$$
\begin{aligned}
|C| & =\left|\cup_{i} \mathcal{B}\left(X_{i}, 5 r_{X_{i}}\right) \cap C\right| \\
& \leq \sum_{i}\left|\mathcal{B}\left(X_{i}, 5 r_{X_{i}}\right) \cap C\right| \\
& \leq \epsilon \sum_{i}\left|\mathcal{B}\left(X_{i}, 5 r_{X_{i}}\right)\right| \\
& \leq \epsilon(5 \gamma)^{2+\sigma} \sum_{i}\left|\mathcal{B}\left(X_{i}, r_{X_{i}}\right)\right| .
\end{aligned}
$$

For every point $X_{i}$, there is a point $Z_{i}$, satisfying $\tilde{d}\left(Z_{i}, X_{i}\right)=\frac{{ }^{r} X_{i}}{4}$ and $\mathcal{B}\left(Z_{i}, \frac{{ }^{r} X_{i}}{4}\right) \subset$ $\mathcal{B}\left(X_{i}, r_{X_{i}}\right) \cap \mathcal{B}_{1}$. Thus,

$$
\begin{aligned}
\frac{\left|\mathcal{B}\left(X_{i}, r_{X_{i}}\right)\right|}{\left|\mathcal{B}\left(X_{i}, r_{X_{i}}\right) \cap \mathcal{B}_{1}\right|} & \leq \frac{\left|\mathcal{B}\left(X_{i}, r_{X_{i}}\right)\right|}{\left|\mathcal{B}\left(Z_{i}, \frac{r_{X_{i}}}{4}\right)\right|} \leq \frac{\left|B\left(X_{i}, \alpha_{2} r_{X_{i}}\right)\right|}{\left|B\left(Z_{i}, \frac{1}{4} \alpha_{1} r_{X_{i}}\right)\right|} \\
& \leq \frac{\left|B\left(Z_{i}, \alpha r_{X_{i}}\right)\right|}{\left|B\left(Z_{i}, \alpha_{1} r_{X_{i}}\right)\right|} \leq\left(\frac{5}{4} \gamma^{2}\right)^{2+\sigma},
\end{aligned}
$$

where $\alpha=\frac{5 \alpha_{2}^{2}}{4 \alpha_{1}}$.

By the above inequalities we have

$$
|C| \leq \epsilon\left(\frac{25}{4} \gamma^{3}\right)^{2+\sigma} \sum_{i}\left|\mathcal{B}\left(X_{i}, r_{X_{i}}\right) \cap \mathcal{B}_{1}\right| \leq \epsilon\left(7 \gamma^{3}\right)^{2+\sigma}|D| .
$$

So the proof is finished.

Similarly we have the following equivalent lemma. 
Lemma 2.4. Let $0<\epsilon<1, C \subset D \subset B_{1}$ be two measurable sets contained in $B_{1}$ with $|C|<\epsilon\left|B_{1}\right|$. Assume for every $X \in C$ and $r<2 \gamma$, if $|C \cap B(X, r)| \geq$ $\epsilon|B(X, r)|$, then $B(X, r) \cap B_{1} \subset D$. Then $|C| \leq C(\gamma) \epsilon|D|$.

Remark. Here we use the Lebesgue differentiation theorem of the characteristic function $\chi_{C}$ with the Euclidean balls replaced by the balls we defined, but this is easily seen by using the same method as in [13].

For the Maximal function, the weak $(1,1)$ and the strong $(p, p)$ estimates hold.

\section{Lemma 2.5 .}

(1) If $f \in L^{1}(\Omega)$, then for every $\lambda>0$,

$$
|\{X \in \Omega: \mathcal{M} f>\lambda\}| \leq \frac{C(\gamma)}{\lambda}\|f\|_{L^{1}(\Omega)} .
$$

(2) If $f \in L^{p}(\Omega), 1<p \leq \infty$, then

$$
\|\mathcal{M} f\|_{L^{p}(\Omega)} \leq C(\gamma, p)\|f\|_{L^{p}(\Omega)} .
$$

\section{Spaces associated with the vector fields}

In order to study the weak solutions we introduce the Sobolev-type spaces which associate with the vector fields $\partial x,|x|^{\sigma} \partial y$. For $1 \leq p<\infty$, we define the function space

$$
W_{*}^{1, p}(\Omega):=\left\{u \in L^{p}(\Omega) ; u_{x} \in L^{p}(\Omega),|x|^{\sigma} u_{y} \in L^{p}(\Omega)\right\} .
$$

Then $W_{*}^{1, p}(\Omega)$ is a Banach space with the norm defined by

$$
\left.\left\|\left.u\right|_{W_{*}^{1, p}(\Omega)}=\right\| u\left\|_{L^{p}(\Omega)}+\right\| u_{x}\left\|_{L^{p}(\Omega)}+\right\||| x\right|^{\sigma} u_{y} \|_{L^{p}(\Omega)} .
$$

Another Banach space $W_{0, *}^{1, p}(\Omega)$ arises by taking the closure of $C_{0}^{\infty}(\Omega)$ in $W_{*}^{1, p}(\Omega)$. In particularly, we denote $W_{*}^{1,2}(\Omega), W_{0, *}^{1,2}(\Omega)$ by $H_{*}^{1}(\Omega), H_{0, *}^{1}(\Omega)$.

The imbedding results and Poincaré inequalities for the vector fields have been studied in many papers, for instance, $[2,3,6,8,10,11]$. Here we need the following results.

By $[14$, Corollary 1] we have

Lemma 3.1. For any $\sigma>0$, there is a small constant $h=h(\sigma)>0$, so that for any $r<R \leq 1$, there is a $C_{\sigma}(r, R)>0$, such that

$$
\|u\|_{H^{h}\left(B_{r}\right)} \leq C_{\sigma}(r, R)\|u\|_{H_{*}^{1}\left(B_{R}\right)} .
$$


Let

$$
\bar{u}_{\Omega}=\frac{1}{|\Omega|} \int_{\Omega} u(x, y) d x d y
$$

be the average of $u(x, y)$ over $\Omega$.

Then the following Poincaré type inequality holds (see $[3,5])$.

Lemma 3.2. If $u \in H_{*}^{1}(B(X, r))$, then

$$
\int_{B(X, r)}\left|u-\bar{u}_{B(X, r)}\right|^{2} d x d y \leq C r^{2} \int_{B(X, r)}\left|u_{x}\right|^{2}+|x|^{2 \sigma}\left|u_{y}\right|^{2} d x d y .
$$

In [3] the author used the intrinsic metric which is called Carnot-Carathéodory metric to prove the Sobolev-Poincaré type inequality. For $p \geq 1$, here we use the classical Poincaré inequality to give a very simple proof of the degenerate one, which works for all $\sigma \geq 0$.

Lemma 3.2' Let $Q^{r}=(-2 r, 2 r) \times\left(-r^{1+\sigma}, r^{1+\sigma}\right), 1 \leq p<\infty$. If $u \in W_{*}^{1, p}\left(Q^{r}\right)$, then there exists a constant $C$ depending on $p$ and $\sigma$, such that

$$
\int_{Q^{r}}\left|u-\bar{u}_{Q^{r}}\right|^{p} \leq C r^{p} \int_{Q^{r}}\left|u_{x}\right|^{p}+|x|^{\sigma p}\left|u_{y}\right|^{p} d x d y .
$$

Proof. By scaling, we need only to prove it for $r=1$. For $(x, y) \in Q^{1}$, we have

$$
|x|^{\sigma} \geq \frac{1}{2}|x|^{[\sigma]+1}
$$

where $[\sigma]$ is the integer part of $\sigma$. Therefore we need only to prove the lemma when $\sigma$ is a positive integer. In what follows we assume $\sigma \in \mathbb{N}$ and $r=1$.

Let

$$
\begin{aligned}
Q_{1} & =(0,2) \times(-1,1), \\
Q_{n, 0} & =\left(2^{-(n-1)}, 2^{-(n-2)}\right) \times(-1,1), \quad n=1,2, \ldots
\end{aligned}
$$

We divide each $Q_{n, 0}$ into $2^{(n-1)(\sigma+1)}$ cubes and denote them by $Q_{n, 1}, Q_{n, 2}, \ldots$, $Q_{n, 2^{(n-1)(\sigma+1)}}$ from up to down. Thus,

$$
Q_{1}=\cup_{n=1}^{\infty} Q_{n, 0} \cup A=\cup_{(n, m)} Q_{n, m} \cup \tilde{A},
$$

where $A$ and $\tilde{A}$ are the sets of measure zero. 
For every cube $Q_{n, m}$ there exists a point $\left(x_{n, m}, y_{n, m}\right)$ such that

$$
Q_{n, m}=\left(2^{-(n-1)}, 2^{-(n-2)}\right) \times\left(y_{n, m}-2^{-(n-1)(\sigma+1)}, y_{n, m}+2^{-(n-1)(\sigma+1)}\right) .
$$

For each cube $Q_{n, l}, n \geq 2$, there exists a cube $Q_{n-1, l_{1}}$ such that the right side boundary of $Q_{n, l}$, i.e.,

$$
2^{-(n-2)} \times\left[y_{n, l}-2^{-(n-1)(\sigma+1)}, y_{n, l}+2^{-(n-1)(\sigma+1)}\right],
$$

is a subset of the left side boundary of $Q_{n-1, l_{1}}$, i.e.,

$$
2^{-(n-2)} \times\left[y_{n-1, l_{1}}-2^{-(n-2)(\sigma+1)}, y_{n-1, l_{1}}+2^{-(n-2)(\sigma+1)}\right],
$$

and it is clear that $\left(l_{1}-1\right) 2^{\sigma+1}<l \leq l_{1} \cdot 2^{\sigma+1}$. If the two cubes have the above relationship, then denote

$$
Q_{n, l} \propto Q_{n-1, l_{1}} .
$$

Thus, for each cube $Q_{n, m}$, there exists a sequence $\left\{Q_{n-k, m_{k}}\right\}_{k=1}^{n-1}$ such that

$$
Q_{n, m} \propto Q_{n-1, m_{1}} \propto \cdots \propto Q_{1,1},
$$

and also there exists a sequence $\left\{Q_{n+k, m_{k}}\right\}_{k=1}^{\infty}$ such that

$$
\cdots \propto Q_{n+k, m_{k}} \propto \cdots \propto Q_{n+1, m_{1}} \propto Q_{n, m} .
$$

We denote

$$
(n, m) \propto(k, l),
$$

if there exists a sequence $\left\{Q_{n_{i}, m_{i}}\right\}_{i=n-1}^{n-k-1}$ such that

$$
Q_{n, m} \propto Q_{n-1, m_{1}} \propto \cdots \propto Q_{k, l} .
$$

To prove the lemma we give some notations:

The set $\{(n, m):(n, m) \propto(k, l)\}$ means the pair of numbers $(k, l)$ is fixed and all $(n, m)$ satisfying the relation $(n, m) \propto(k, l)$ are included, so does the set $\{(n, m):(k, l) \propto(n, m)\}$;

The set $\{m:(n, m) \propto(k, l)\}$ means $(k, l)$ and $n$ are fixed and all $(n, m)$ satisfying the relation $(n, m) \propto(k, l)$ are included.

Obviously,

$\bigcup_{\{(n, m):(n, m) \propto(k, l)\}} Q_{n, m}=\left(0,2^{-(k-1)}\right) \times\left(y_{k, l}-2^{-(k-1)(\sigma+1)}, y_{k, l}+2^{-(k-1)(\sigma+1)}\right) \backslash B$, 
where $B$ is a set of measure zero, so the measures of the sets satisfy

$$
\left|\cup_{\{(n, m):(k, l) \propto(n, m)\}} Q_{n, m}\right|=\left|Q_{k, l}\right|,
$$

and one can also easily have

$$
\sum_{\{m:(n, m) \propto(k, l)\}}\left|Q_{n, m}\right|=2^{k-n}\left|Q_{k, l}\right|
$$

In each cube $Q_{n, m}$, let

$$
v(x, y)=u\left(2^{-(n-1)} x, 2^{-(\sigma+1)(n-1)} y+y_{n, m}\right) \quad(x, y) \in Q_{1,1} .
$$

By the classical Poincaré inequality,

$$
\int_{Q_{1,1}}\left|v-\bar{v}_{Q_{1,1}}\right|^{p} \leq C \int_{Q_{1,1}}\left|v_{x}\right|^{p}+\left|v_{y}\right|^{p} d x d y .
$$

For any point $(x, y) \in Q_{1,1}$, we have $|x| \geq 1$, so

$$
\int_{Q_{1,1}}\left|v-\bar{v}_{Q_{1,1}}\right|^{p} \leq C \int_{Q_{1,1}}\left|v_{x}\right|^{p}+|x|^{\sigma p}\left|v_{y}\right|^{p} d x d y
$$

Now scaling back,

$$
\int_{Q_{n, m}}\left|u-\bar{u}_{Q_{n, m}}\right|^{p} d x d y \leq C 2^{-(n-1) p} \int_{Q_{n, m}}\left|u_{x}\right|^{p}+|x|^{\sigma p}\left|u_{y}\right|^{p} d x d y .
$$

For every $Q_{2, i} \propto Q_{1,1}$, there is a constant $C$ depending only on $\sigma$ such that

$$
\left|\bar{u}_{Q_{2, i}}-\bar{u}_{Q_{1,1} \mid}\right| \leq C \frac{1}{\left|Q_{2, i} \cup Q_{1,1}\right|} \int_{Q_{2, i} \cup Q_{1,1}}\left|u_{x}\right|+|x|^{\sigma}\left|u_{y}\right| d x d y .
$$

For $Q_{n, i} \propto Q_{n-1, i_{1}}$, by the scaling form of (3.1), we have

$$
\left|\bar{u}_{Q_{n, i}}-\bar{u}_{Q_{n-1, i_{1}}}\right| \leq C \frac{2^{-(n-1)}}{\left|Q_{n, i} \cup Q_{n-1, i_{1}}\right|} \int_{Q_{n, i} \cup Q_{n-1, i_{1}}}\left|u_{x}\right|+|x|^{\sigma}\left|u_{y}\right| d x d y .
$$


In $Q_{1}$ we give the following estimate,

$$
\begin{aligned}
& \int_{Q_{1}}\left|u(x, y)-\bar{u}_{Q_{1}}\right|^{p} d x d y=\sum_{n=1}^{\infty} \sum_{m=1}^{2^{(\sigma+1)(n-1)}} \int_{Q_{n, m}}\left|u(x, y)-\bar{u}_{Q_{1}}\right|^{p} d x d y \\
& \leq \sum_{n, m} \int_{Q_{n, m}}\left(\left|u(x, y)-\bar{u}_{Q_{n, m}}\right|+\left|\bar{u}_{Q_{n, m}}-\bar{u}_{Q_{1}}\right|\right)^{p} d x d y \\
& \leq 2^{p-1} \sum_{n, m}\left(\int_{Q_{n, m}}\left|u(x, y)-\bar{u}_{Q_{n, m}}\right|^{p} d x d y+\left|Q_{n, m}\right|\left|\bar{u}_{Q_{n, m}}-\bar{u}_{Q_{1}}\right|^{p}\right) \\
& \leq C_{n, p} \sum_{n, m} \int_{Q_{n, m}}\left(\left|u_{x}\right|^{p}+|x|^{\sigma p}\left|u_{y}\right|^{p}\right) d x d y \\
& +2^{p-1} \sum_{n, m}\left|Q_{n, m}\right|\left(\sum_{\{(k, l):(n, m) \propto(k, l)\}}\left|\bar{u}_{Q_{k+1, l_{1}}}-\bar{u}_{Q_{k, l}}\right|+\left|\bar{u}_{Q_{1,1}}-\bar{u}_{Q_{1}}\right|\right)^{p} \\
& \leq C \int_{Q_{1}}\left(\left|u_{x}\right|^{p}+|x|^{\sigma p}\left|u_{y}\right|^{p}\right) d x d y \\
& +4^{p-1} \sum_{n, m}\left|Q_{n, m}\right|\left(\sum_{\{(k, l):(n, m) \propto(k, l)\}} \mid \bar{u}_{Q_{k+1, l_{1}}}-\bar{u}_{Q_{k, l} \mid}\right)^{p}+\left|Q_{1}\right|\left|\bar{u}_{Q_{1,1}}-\bar{u}_{Q_{1}}\right|^{p} \\
& \leq C \int_{Q_{1}}\left(\left|u_{x}\right|^{p}+|x|^{\sigma p}\left|u_{y}\right|^{p}\right) d x d y \\
& +C \sum_{n, m}\left|Q_{n, m}\right| n^{p-1} \sum_{\{(k, l):(n, m) \propto(k, l)\}} \frac{2^{-k p}}{\left|Q_{k+1, l_{1}} \cup Q_{k, l}\right|} \int_{Q_{k+1, l_{1}} \cup Q_{k, l}}\left(\left|u_{x}\right|^{p}\right. \\
& \left.+|x|^{\sigma p}\left|u_{y}\right|^{p}\right) d x d y+C\left|Q_{1}\right|\left(\frac{1}{\left|Q_{1}\right|} \int_{Q_{1}}\left|u_{x}\right| d x d y\right)^{p} \\
& \leq C \int_{Q_{1}}\left(\left|u_{x}\right|^{p}+|x|^{\sigma p}\left|u_{y}\right|^{p}\right) d x d y \\
& +C \sum_{k, l} \sum_{\{(n, m):(n, m) \propto(k, l)\}} \frac{n^{p-1}\left|Q_{n, m}\right| 2^{-k p}}{\left|Q_{k+1, l_{1}} \cup Q_{k, l}\right|} \int_{Q_{k+1, l_{1}} \cup Q_{k, l}}\left(\left|u_{x}\right|^{p}+|x|^{\sigma p}\left|u_{y}\right|^{p}\right) d x d y \\
& \leq C \int_{Q_{1}}\left(\left|u_{x}\right|^{p}+|x|^{\sigma p}\left|u_{y}\right|^{p}\right) d x d y \text {. }
\end{aligned}
$$

By the symmetric property we have

$$
\int_{Q_{2}}\left|u_{x}-\bar{u}_{Q_{2}}\right|^{p} d x d y \leq C \int_{Q_{2}}\left(\left|u_{x}\right|^{p}+|x|^{\sigma p}\left|u_{y}\right|^{p}\right) d x d y,
$$

where $Q_{2}=(-2,0) \times(-1,1)$. 
Therefore,

$$
\begin{aligned}
& \int_{Q^{1}}\left|u-\bar{u}_{Q^{1}}\right|^{p}=\int_{Q_{1}}\left|u-\bar{u}_{Q^{1}}\right|^{p}+\int_{Q_{2}}\left|u-\bar{u}_{Q^{1}}\right|^{p} \\
& \leq C\left(\int_{Q_{1}}\left|u-\bar{u}_{Q_{1}}\right|^{p}+\int_{Q_{2}}\left|u-\bar{u}_{Q_{2}}\right|^{p}+\left|Q_{1}\right|\left|\bar{u}_{Q_{1}}-\bar{u}_{Q^{1}}\right|^{p}+\left|Q_{2}\right|\left|\bar{u}_{Q_{2}}-\bar{u}_{Q^{1}}\right|^{p}\right) \\
& \leq C \int_{Q^{1}}\left|u_{x}\right|^{p}+|x|^{\sigma p}\left|u_{y}\right|^{p} d x d y .
\end{aligned}
$$

This finishes the proof.

\section{Proof of the $L^{p}$ estimates}

Since the equation (1.1) is elliptic away from $x=0$ and translation invariant along the $y$ axis, we start with considering the properties near the point $(x, y)=(0,0)$.

Theorem 4.1. Let $1<p<\infty$. If $u$ is a weak solution of equation (1.1) in $\Omega \supset$ $B_{80 \gamma^{6}{ }_{r}}$, then $u \in W_{*}^{1, p}\left(B_{r}\right)$ with the estimate

$$
\int_{B_{r}}\left(\left|u_{x}\right|^{p}+\left.\left.|| x\right|^{\sigma} u_{y}\right|^{p}\right) d x d y \leq C \int_{B_{5 r}}\left(r^{-p}|u|^{p}+|f|^{p}+|g|^{p}\right) d x d y .
$$

Before we come to the proof of the theorem we give some lemmas.

Lemma 4.2. If $u$ is a weak solution of equation (1.1) and $B_{5} \subset \Omega$, then

$$
\int_{B_{4}}\left(\left|u_{x}\right|^{2}+|x|^{2 \sigma}\left|u_{y}\right|^{2}\right) d x d y \leq C \int_{B_{5}}\left(|u|^{2}+|f|^{2}+|g|^{2}\right) d x d y,
$$

for some universal constant $C$.

Proof. For every $\eta \in C_{0}^{\infty}\left(B_{5}\right)$, take $\eta^{2} u$ as a test function, then

$$
\int_{B_{5}}\left(u_{x}\left(\eta^{2} u\right)_{x}+|x|^{2 \sigma} u_{y}\left(\eta^{2} u\right)_{y}\right) d x d y=\int_{B_{5}}\left(f\left(\eta^{2} u\right)_{x}+|x|^{\sigma} g\left(\eta^{2} u\right)_{y}\right) d x d y .
$$

Thus,

$$
\begin{aligned}
& \int_{B_{5}}\left(\eta^{2}\left|u_{x}\right|^{2}+|x|^{2 \sigma} \eta^{2}\left|u_{y}\right|^{2}\right) d x d y \\
& =-2 \int_{B_{5}}\left(\eta u \eta_{x} u_{x}+|x|^{2 \sigma} \eta u \eta_{y} u_{y}\right)+\int_{B_{5}} f\left(2 \eta u \eta_{x}+\eta^{2} u_{x}\right)+|x|^{\sigma} g\left(2 \eta u \eta_{y}+\eta^{2} u_{y}\right) \\
& \leq \int_{B_{5}} \frac{1}{2} \eta^{2}\left(\left|u_{x}\right|^{2}+|x|^{2 \sigma}\left|u_{y}\right|^{2}\right)+5|u|^{2}\left(\left|\eta_{x}\right|^{2}+|x|^{2 \sigma}\left|\eta_{y}\right|^{2}\right)+\int_{B_{5}} 2|\eta|^{2}\left(|f|^{2}+|g|^{2}\right) .
\end{aligned}
$$


So

$$
\begin{aligned}
\int_{B_{5}} \eta^{2}\left(\left|u_{x}\right|^{2}+|x|^{2 \sigma}\left|u_{y}\right|^{2}\right) d x d y \leq & 10 \int_{B_{5}}|u|^{2}\left(\left|\eta_{x}\right|^{2}+|x|^{2 \sigma}\left|\eta_{y}\right|^{2}\right) d x d y \\
& +4 \int_{B_{5}}\left(|f|^{2}+|g|^{2}\right)|\eta|^{2} d x d y .
\end{aligned}
$$

Now if we take $\eta$ such that

$$
\begin{cases}0 \leq \eta \leq 1 & (x, y) \in B_{5} \\ \eta(x, y)=1 & (x, y) \in B_{4} \\ \eta(x, y)=0 & (x, y) \in R^{n} \backslash B_{5}\end{cases}
$$

and $\left|\eta_{x}\right|,\left|\eta_{y}\right|<C$, then the lemma follows.

Lemma 4.3. For any $\epsilon>0$, there exists a $\delta>0$, so that if $u$ is a weak solution of (1.1) in $B_{6}$ with the conditions that

$$
\begin{aligned}
& \frac{1}{\left|B_{6}\right|} \int_{B_{6}}\left(\left|u_{x}\right|^{2}+|x|^{2 \sigma}\left|u_{y}\right|^{2}\right) d x d y \leq 1, \\
& \frac{1}{\left|B_{6}\right|} \int_{B_{6}}\left(|f(x, y)|^{2}+|g(x, y)|^{2}\right) d x d y \leq \delta,
\end{aligned}
$$

then

$$
\frac{1}{\left|B_{5}\right|} \int_{B_{5}}|u-v|^{2} d x d y \leq \epsilon,
$$

for some $v(x, y)$ which is a solution of $\mathcal{L} v(x, y)=0$ in $B_{5}$.

Proof. We prove it by contradiction. Suppose there exists an $\epsilon_{0}>0$, such that for any $\frac{1}{n}$, there exist $u_{n}, f_{n}$, and $g_{n}$ satisfying

$$
\begin{aligned}
& \frac{1}{\left|B_{6}\right|} \int_{B_{6}}\left(\left|\left(u_{n}\right)_{x}\right|^{2}+|x|^{2 \sigma}\left|\left(u_{n}\right)_{y}\right|^{2}\right) d x d y \leq 1, \\
& \frac{1}{\left|B_{6}\right|} \int_{B_{6}}\left(\left|f_{n}(x, y)\right|^{2}+\left|g_{n}(x, y)\right|^{2}\right) d x d y \leq \frac{1}{n},
\end{aligned}
$$

and

$$
\left(u_{n}\right)_{x x}+|x|^{2 \sigma}\left(u_{n}\right)_{y y}=\left(f_{n}\right)_{x}+|x|^{\sigma}\left(g_{n}\right)_{y}
$$

in the weak sense in $B_{6}$, but for any solution of equation $\mathcal{L} v=0$ in $B_{5}$, we have

$$
\frac{1}{\left|B_{5}\right|} \int_{B_{5}}\left|u_{n}-v\right|^{2} d x d y \geq \epsilon_{0} .
$$


Since the equation is linear, we may assume $\left\|u_{n}\right\|_{L^{2}\left(B_{6}\right)} \leq 1$. Thus by Lemma 3.1,

$$
\left\|u_{n}\right\|_{H^{h}\left(B_{5}\right)} \leq C\left(\left\|\left(u_{n}\right)_{x}\right\|_{L^{2}\left(B_{6}\right)}+\left\||x|^{\sigma}\left(u_{n}\right)_{y}\right\|_{L^{2}\left(B_{6}\right)}+\left\|u_{n}\right\|_{L^{2}\left(B_{6}\right)}\right) \leq C .
$$

Since $H^{h}$ is compactly embedded in $L^{2}$, there is a subsequence of $u_{n}$, which we still denote as $u_{n}$, such that

$$
u_{n} \rightarrow v \quad \text { strongly in } L^{2}\left(B_{5}\right) .
$$

By the $L^{2}$ boundedness of $\left(u_{n}\right)_{x}$ and $|x|^{\sigma}\left(u_{n}\right)_{y}$ we have

$$
\left(u_{n}\right)_{x} \rightarrow v_{x} \quad \text { weakly in } L^{2}\left(B_{5}\right),
$$

and

$$
|x|^{\sigma}\left(u_{n}\right)_{y} \rightarrow|x|^{\sigma} v_{y} \quad \text { weakly in } L^{2}\left(B_{5}\right) .
$$

Since $u_{n}$ is a weak solution, we have

$$
\left.\int_{B_{5}}\left(u_{n}\right)_{x} \varphi_{x}+|x|^{2 \sigma}\left(u_{n}\right)_{y} \varphi_{y}\right) d x d y=\int_{B_{5}}\left(f_{n} \varphi_{x}+|x|^{\sigma} g_{n} \varphi_{y}\right) d x d y,
$$

for any $\varphi \in H_{0, *}^{1}\left(B_{5}\right)$. Now let $n \rightarrow \infty$, we have

$$
\int_{B_{5}}\left(v_{x} \varphi_{x}+|x|^{2 \sigma} v_{y} \varphi_{y}\right) d x d y=0,
$$

which is a contradiction. So the proof is finished.

A consequence of Lemma 4.2 and 4.3 is the following:

Lemma 4.4. For any $\epsilon>0$, there exists $a \delta>0$ so that if $u$ is a weak solution of equation (1.1) with the conditions that

$$
\begin{aligned}
& \frac{1}{\left|B_{6}\right|} \int_{B_{6}}\left(\left|u_{x}\right|^{2}+|x|^{2 \sigma}\left|u_{y}\right|^{2}\right) d x d y \leq 1, \\
& \frac{1}{\left|B_{6}\right|} \int_{B_{6}}\left(|f(x, y)|^{2}+|g(x, y)|^{2}\right) d x d y \leq \delta,
\end{aligned}
$$

then there exists a weak solution $v$ of $\mathcal{L} v=0$ in $B_{4}$ such that

$$
\int_{B_{4}}\left(\left|u_{x}-v_{x}\right|^{2}+|x|^{2 \sigma}\left|u_{y}-v_{y}\right|^{2}\right) d x d y \leq \epsilon^{2} .
$$

Before we state an important lemma, we shall introduce the following regularity result for the homogeneous equation. See [14, Lemma 2]. 
Lemma 4.5. If $\mathcal{L} v(x, y)=0$ in $B_{1}$, then $v$ is $C^{2,2 \sigma}$ in $B_{\frac{1}{2}}$, and

$$
\|v\|_{C^{2,2 \sigma}\left(B_{\frac{1}{2}}\right) \leq C(\sigma)\|v\|_{L^{2}\left(B_{1}\right)}} .
$$

Lemma 4.6. There is a constant $N_{1}$, so that for any $\epsilon>0$, there exists a $\delta>0$, such that the following statement holds: if $u$ is a weak solution of (1.1) in $\Omega \supset B_{8 \gamma^{4}}$, with

$$
\left\{\mathcal{M}\left(|f|^{2}+|g|^{2}\right) \leq \delta^{2}\right\} \cap\left\{\mathcal{M}\left(\left|u_{x}\right|^{2}+|x|^{2 \sigma}\left|u_{y}\right|^{2}\right) \leq 1\right\} \cap B_{1} \neq \emptyset,
$$

then

$$
\left|\left\{\mathcal{M}\left(\left|u_{x}\right|^{2}+|x|^{2 \sigma}\left|u_{y}\right|^{2}\right)>N_{1}^{2}\right\} \cap B_{1}\right|<\epsilon\left|B_{1}\right| .
$$

Proof. From (4.2), there is a point $X_{0} \in B_{1}$, such that

$$
\frac{1}{\left|B\left(X_{0}, r\right)\right|} \int_{B\left(X_{0}, r\right) \cap \Omega}\left(\left|u_{x}\right|^{2}+|x|^{2 \sigma}\left|u_{y}\right|^{2}\right) d x d y \leq 1
$$

and

$$
\frac{1}{\left|B\left(X_{0}, r\right)\right|} \int_{B\left(X_{0}, r\right) \cap \Omega}\left(|f(x, y)|^{2}+|g(x, y)|^{2}\right) d x d y \leq \delta^{2} .
$$

Since $B_{6 \gamma^{2}} \subset B_{7 \gamma^{3}}\left(X_{0}\right) \subset B_{8 \gamma^{4}}$, we have

$$
\frac{1}{\left|B_{6 \gamma^{2}}\right|} \int_{B_{6 \gamma^{2}}}\left(\left|u_{x}\right|^{2}+|x|^{2 \sigma}\left|u_{y}\right|^{2}\right) d x d y \leq\left(2 \gamma^{2}\right)^{2+\sigma},
$$

and

$$
\frac{1}{\left|B_{6 \gamma^{2}}\right|} \int_{B_{6 \gamma^{2}}}\left(|f(x, y)|^{2}+|g(x, y)|^{2}\right) d x d y \leq\left(2 \gamma^{2}\right)^{2+\sigma} \delta^{2} .
$$

By Lemma 4.4, for any $\eta>0$ (here we take $\eta<1$ ), there exists a $\delta$ depending on $\eta$, such that

$$
\int_{B_{4 \gamma^{2}}}\left(\left|u_{x}-v_{x}\right|^{2}+|x|^{2 \sigma}\left|u_{y}-v_{y}\right|^{2}\right) d x d y \leq \eta^{2},
$$

where $v(x, y)$ is a weak solution of $\mathcal{L} v(x, y)=0$ in $B_{4 \gamma^{2}}$. Thus by the inequalities (4.4) and (4.6),

$$
\int_{B_{4 \gamma^{2}}}\left(\left|v_{x}\right|^{2}+|x|^{2 \sigma}\left|v_{y}\right|^{2}\right) d x d y \leq 2\left(1+\left(2 \gamma^{2}\right)^{2+\sigma}\left|B_{6 \gamma^{2}}\right|\right) .
$$

Let $\tilde{v}(x, y)=v(x, y)-\bar{v}_{B_{4 \gamma^{2}}}$, then $\tilde{v}(x, y)$ satisfies $\mathcal{L} \tilde{v}(x, y)=0$ and the same estimate as $v$ in inequality (4.7). By the Poincaré type inequality and Lemma 4.5, there is a constant $N_{0}$ such that

$$
\left\|\tilde{v}_{x}\right\|_{L^{\infty}\left(B_{3 \gamma^{2}}\right)}+\left\||x|^{\sigma} \tilde{v}_{y}\right\|_{L^{\infty}\left(B_{3 \gamma^{2}}\right)} \leq N_{0}
$$


therefore,

$$
\left\|v_{x}\right\|_{L^{\infty}\left(B_{\left.3 \gamma^{2}\right)}\right.}+\left\||x|^{\sigma} v_{y}\right\|_{L^{\infty}\left(B_{\left.3 \gamma^{2}\right)}\right.} \leq N_{0} .
$$

By (4.6) and Lemma 2.5,

$$
\begin{aligned}
& \left|\left\{X \in B_{3 \gamma^{2}}: \mathcal{M}_{B_{3 \gamma^{2}}}\left(\left|u_{x}-v_{x}\right|^{2}+|x|^{2 \sigma}\left|u_{y}-v_{y}\right|^{2}\right)>N_{0}^{2}\right\}\right| \\
& \leq \frac{C(\gamma)}{N_{0}^{2}} \int_{B_{3 \gamma^{2}}}\left(\left|u_{x}-v_{x}\right|^{2}+|x|^{2 \sigma}\left|u_{y}-v_{y}\right|^{2}\right) d x d y \\
& \leq \frac{C(\gamma)}{N_{0}^{2}} \eta^{2} .
\end{aligned}
$$

Now let $X_{1}$ be a point in $\left\{\mathcal{M}_{B_{3 \gamma^{2}}}\left(\left(u_{x}-v_{x}\right)^{2}+|x|^{2 \sigma}\left(u_{y}-v_{y}\right)^{2}\right) \leq N_{0}^{2}\right\} \cap B_{1}$.

If $r \leq 2 \gamma$, then $B\left(X_{1}, r\right) \subset B_{3 \gamma^{2}}$. So

$$
\begin{aligned}
& \frac{1}{\left|B\left(X_{1}, r\right)\right|} \int_{B\left(X_{1}, r\right)}\left(\left|u_{x}\right|^{2}+|x|^{2 \sigma}\left|u_{y}\right|^{2}\right) d x d y \\
& \leq \frac{2}{\left|B\left(X_{1}, r\right)\right|} \int_{B\left(X_{1}, r\right)}\left(\left|u_{x}-v_{x}\right|^{2}+|x|^{2 \sigma}\left|u_{y}-v_{y}\right|^{2}\right) d x d y \\
& \quad+\frac{2}{\left|B\left(X_{1}, r\right)\right|} \int_{B\left(X_{1}, r\right)}\left(\left|v_{x}\right|^{2}+|x|^{2 \sigma}\left|v_{y}\right|^{2}\right) d x d y \\
& \leq 4 N_{0}^{2} .
\end{aligned}
$$

If $r>2 \gamma$, then $B\left(X_{1}, r\right) \subset B\left(X_{0}, 2 \gamma r\right)$ and

$$
\begin{aligned}
& \frac{1}{\left|B\left(X_{1}, r\right)\right|} \int_{B\left(X_{1}, r\right)}\left(\left|u_{x}\right|^{2}+|x|^{2 \sigma}\left|u_{y}\right|^{2}\right) d x d y \\
& \leq \frac{\left|B\left(X_{0}, 2 \gamma r\right)\right|}{\left|B\left(X_{1}, r\right)\right|} \frac{1}{\left|B\left(X_{0}, 2 \gamma r\right)\right|} \int_{B\left(X_{0}, 2 \gamma r\right)}\left(\left|u_{x}\right|^{2}+|x|^{2 \sigma}\left|u_{y}\right|^{2}\right) d x d y \\
& \leq \frac{\left|B\left(X_{1}, \gamma(2 \gamma+1) r\right)\right|}{\left|B\left(X_{1}, r\right)\right|} \\
& \leq(\gamma(2 \gamma+1))^{2+\sigma} .
\end{aligned}
$$

Therefore, if we take $N_{1}^{2}=\max \left\{4 N_{0}^{2},(\gamma(2 \gamma+1))^{2+\sigma}\right\}$, then

$$
\left\{\mathcal{M}\left(\left|u_{x}\right|^{2}+|x|^{2 \sigma}\left|u_{y}\right|^{2}\right)>N_{1}^{2}\right\} \cap B_{1} \subset\left\{\mathcal{M}_{B_{3 \gamma^{2}}}\left(\left|u_{x}-v_{x}\right|^{2}+|x|^{2 \sigma}\left|u_{y}-v_{y}\right|^{2}\right)>N_{0}^{2}\right\} \cap B_{1} \text {. }
$$

From (4.8) we have

$$
\begin{aligned}
& \left|\left\{\mathcal{M}\left(\left|u_{x}\right|^{2}+|x|^{2 \sigma}\left|u_{y}\right|^{2}\right)>N_{1}^{2}\right\} \cap B_{1}\right| \\
& \leq\left|\left\{\mathcal{M}_{B_{3 \gamma^{2}}}\left(\left|u_{x}-v_{x}\right|^{2}+|x|^{2 \sigma}\left|u_{y}-v_{y}\right|^{2}\right)>N_{0}^{2}\right\} \cap B_{1}\right| \\
& \leq \frac{C(\gamma)}{N_{0}^{2}} \eta^{2}<\epsilon\left|B_{1}\right|
\end{aligned}
$$

by taking $\eta$ small enough and hence the lemma is proved. 
Corollary 4.7. There is a constant $N_{1}$, so that for any $\epsilon>0$ there exists a $\delta>0$ such that the following statements hold.

1. Take $Y=(0, y)$. If $u$ is a weak solution of $(1.1)$ in $\Omega \supset B\left(Y, 8 \gamma^{4} r\right)$ and

$$
\left\{\mathcal{M}\left(|f|^{2}+|g|^{2}\right) \leq \delta^{2}\right\} \cap\left\{\mathcal{M}\left(\left|u_{x}\right|^{2}+|x|^{2 \sigma}\left|u_{y}\right|^{2}\right) \leq 1\right\} \cap B(Y, r) \neq \emptyset,
$$

then

$$
\left|\left\{\mathcal{M}\left(\left|u_{x}\right|^{2}+|x|^{2 \sigma}\left|u_{y}\right|^{2}\right)>N_{1}^{2}\right\} \cap B(Y, r)\right|<\epsilon|B(Y, r)| .
$$

2. If $u$ is a weak solution of (1.1) in $\Omega \supset B_{80 \gamma^{6}}$, then for any point $X \in B_{1}$ and every $0<r<2 \gamma$, if

$$
\left|\left\{\mathcal{M}\left(\left|u_{x}\right|^{2}+|x|^{2 \sigma}\left|u_{y}\right|^{2}\right)>N_{1}^{2}\right\} \cap B(X, r)\right| \geq \epsilon|B(X, r)|,
$$

then

$$
B(X, r) \subset\left\{\mathcal{M}\left(|f|^{2}+|g|^{2}\right)>\delta^{2}\right\} \cup\left\{\mathcal{M}\left(\left|u_{x}\right|^{2}+|x|^{2 \sigma}\left|u_{y}\right|^{2}\right)>1\right\} .
$$

Proof. Statement 1 is easily seen by translating the equation in $y$ direction and a scaling argument of Lemma 4.6.

We give the proof of statement 2 in the following two cases.

Case 1: $d(B(X, r),\{x=0\}) \leq 18 \gamma^{2} r$. In this case we prove it by contradiction. Let $Y$ be a point on $x=0$ such that

$$
d(Y, B(X, r))=d(B(X, r),\{x=0\}) .
$$

Then,

$$
B(X, r) \subset B\left(Y, 20 \gamma^{3} r\right) \subset B\left(X, 39 \gamma^{4} r\right)
$$

If

$$
\left\{\mathcal{M}\left(|f|^{2}+|g|^{2}\right) \leq \delta^{2}\right\} \cap\left\{\mathcal{M}\left(\left|u_{x}\right|^{2}+|x|^{2 \sigma}\left|u_{y}\right|^{2}\right) \leq 1\right\} \cap B(X, r) \neq \emptyset,
$$

then

$$
\left\{\mathcal{M}\left(|f|^{2}+|g|^{2}\right) \leq \delta^{2}\right\} \cap\left\{\mathcal{M}\left(\left|u_{x}\right|^{2}+|x|^{2 \sigma}\left|u_{y}\right|^{2}\right) \leq 1\right\} \cap B\left(Y, 20 \gamma^{3} r\right) \neq \emptyset .
$$

Taking $\epsilon$ in (4.10) as $\epsilon C_{\gamma}$, where $C_{\gamma}=\left(39 \gamma^{4}\right)^{-(2+\sigma)}$, we have

$$
\left|\left\{\mathcal{M}\left(u_{x}^{2}+|x|^{2 \sigma} u_{y}^{2}\right)>N_{1}^{2}\right\} \cap B\left(Y, 20 \gamma^{3} r\right)\right|<\epsilon C_{\gamma}\left|B\left(Y, 20 \gamma^{3} r\right)\right| .
$$


This implies

$$
\left|\left\{\mathcal{M}\left(\left|u_{x}\right|^{2}+|x|^{2 \sigma}\left|u_{y}\right|^{2}\right)>N_{1}^{2}\right\} \cap B(X, r)\right|<\epsilon|B(X, r)|,
$$

which is a contradiction.

Case 2: $d(B(X, r),\{x=0\})>18 \gamma^{2} r$. We denote the center point of the ball $B(X, r)$ by $X_{0}=\left(x_{0}, y_{0}\right)$. Recall the remark after the definition of the local maximal function (2.3) and let

$$
v(x, y)=\frac{u\left(\left|x_{0}\right| x,\left|x_{0}\right|^{1+\sigma} y\right)}{\left|x_{0}\right|}
$$

then $v(x, y)$ satisfies

$$
\mathcal{L} v=\partial_{x} \tilde{f}+|x|^{\sigma} \partial_{y} \widetilde{g}
$$

where $\tilde{f}(x, y)=f\left(\left|x_{0}\right| x,\left|x_{0}\right|^{1+\sigma} y\right), \widetilde{g}(x, y)=g\left(\left|x_{0}\right| x,\left|x_{0}\right|^{1+\sigma} y\right)$.

Now the corresponding good point of $v(x, y)$ is

$$
\widetilde{X}_{0}=\frac{X_{0}}{\left|x_{0}\right|}= \begin{cases}\left(1, \frac{y_{0}}{\left|x_{0}\right|^{1+\sigma}}\right) & \text { if } \quad x_{0}>0, \\ \left(-1, \frac{y_{0}}{\left|x_{0}\right|^{1+\sigma}}\right) & \text { if } \quad x_{0}<0 .\end{cases}
$$

Since $\left|x_{0}\right|>18 \gamma^{2} r$, consider the equation (4.13) in $B\left(\widetilde{X_{0}}, \frac{6 \gamma^{2} r}{\left|x_{0}\right|}\right)$, then the operator $\mathcal{L}$ is uniformly elliptic, that is

$$
\left(\frac{2}{3}\right)^{2 \sigma}|\xi|^{2} \leq a_{i j}(x, y) \xi_{i} \xi_{j} \leq\left(\frac{4}{3}\right)^{2 \sigma}|\xi|^{2}
$$

for all $\xi=\left(\xi_{1}, \xi_{2}\right) \in \mathbb{R}^{2}$, where $\left[a_{i j}(x, y)\right]$ is the coefficient matrix of $\mathcal{L}$. Since near $\widetilde{X_{0}}$, the coefficients are smooth and the maximal function defined in (2.3) is equivalent to the classical Hardy-Littlewood maximal function, we can use the normal estimates as in [15] to have the following local result:

There exists a constant $N_{0}$, so that for any $\epsilon>0$, there exists a $\delta>0$, such that if

$$
\left\{\mathcal{M}\left(|\widetilde{f}|^{2}+|\widetilde{g}|^{2}\right) \leq \delta^{2}\right\} \cap\left\{\mathcal{M}\left(\left|v_{x}\right|^{2}+|x|^{2 \sigma}\left|v_{y}\right|^{2}\right) \leq 1\right\} \cap B\left(\widetilde{X_{0}}, \frac{r}{\left|x_{0}\right|}\right) \neq \emptyset,
$$

then

$$
\begin{aligned}
& \left|\left\{X \in B\left(\widetilde{X_{0}}, \frac{3 \gamma^{2} r}{\left|x_{0}\right|}\right): \mathcal{M}_{B\left(\widetilde{X_{0}}, \frac{3 \gamma^{2} r}{\left|x_{0}\right|}\right)}\left(\left|v_{x}-w_{x}\right|^{2}+\left|v_{y}-w_{y}\right|^{2}\right)>N_{0}^{2}\right\}\right| \\
& \leq \epsilon\left|B\left(\widetilde{X_{0}}, \frac{r}{\left|x_{0}\right|}\right)\right|,
\end{aligned}
$$


where $w(x, y)$ satisfies

$$
w_{x x}+|x|^{2 \sigma} w_{y y}=0 \quad(x, y) \in B\left(\widetilde{X}_{0}, \frac{4 \gamma^{2} r}{\left|x_{0}\right|}\right),
$$

and

$$
\left\|w_{x}\right\|_{L^{\infty}\left(B\left(\widetilde{X_{0}}, \frac{3 \gamma^{2} r}{\left|x_{0}\right|}\right)\right)}+\left\|w_{y}\right\|_{L^{\infty}\left(B\left(\widetilde{X_{0}}, \frac{3 \gamma^{2} r}{\left|x_{0}\right|}\right)\right)} \leq N_{0}
$$

Furthermore, for each $X \in B\left(\widetilde{X_{0}}, \frac{3 \gamma^{2} r}{\left|x_{0}\right|}\right)$, we have

$$
|| x|-1| \leq \frac{3 \gamma^{2} r}{\left|x_{0}\right|}<\frac{1}{6}
$$

Thus,

$$
\begin{aligned}
& \left|\left\{X \in B\left(\widetilde{X_{0}}, \frac{3 \gamma^{2} r}{\left|x_{0}\right|}\right): \mathcal{M}_{B\left(\widetilde{X_{0}}, \frac{3 \gamma^{3} r}{\left|x_{0}\right|}\right)}\left(\left|v_{x}-w_{x}\right|^{2}+|x|^{2 \sigma}\left|v_{y}-w_{y}\right|^{2}\right) \geq \widetilde{N}_{0}^{2}\right\}\right| \\
& \leq \epsilon\left|B\left(\widetilde{X_{0}}, \frac{r}{\left|x_{0}\right|}\right)\right|,
\end{aligned}
$$

where $\widetilde{N}_{0}=\left(\frac{7}{6}\right)^{\sigma} N_{0}$.

By the definition of the maximal function we have

$$
\mathcal{M} \tilde{f}(X)=\mathcal{M} f\left(\left|x_{0}\right| X\right)
$$

Now, scaling back, we have the local result of $u(x, y)$, that is, if

$$
\left\{\mathcal{M}\left(|f|^{2}+|g|^{2}\right) \leq \delta^{2}\right\} \cap\left\{\mathcal{M}\left(\left|u_{x}\right|^{2}+|x|^{2 \sigma}\left|u_{y}\right|^{2}\right) \leq 1\right\} \cap B\left(X_{0}, r\right) \neq \emptyset
$$

then

$$
\begin{aligned}
& \left|\left\{X \in B\left(X_{0}, 3 \gamma^{2} r\right): \mathcal{M}_{B\left(X_{0}, 3 \gamma^{2} r\right)}\left(\left|u_{x}-\widetilde{w}_{x}\right|^{2}+\left|u_{y}-\widetilde{w}_{y}\right|^{2}\right)>\widetilde{N}_{0}^{2}\right\}\right| \\
& \leq \epsilon\left|B\left(X_{0}, r\right)\right|,
\end{aligned}
$$

where $\tilde{w}(x, y)=\left|x_{0}\right| w\left(\frac{x}{\left|x_{0}\right|}, \frac{y}{\left|x_{0}\right|^{1+\sigma}}\right)$. One can also drop the localization in the inequality (4.15) as in Lemma 4.6 to have the statement 2. The proof is complete.

Corollary 4.8. Assume $u$ is a weak solution of equation (1.1) in a domain $\Omega \supset$ $B_{80 \gamma^{6}}$, with the condition that $\left|\left\{X \in B_{1}: \mathcal{M}\left(\left|u_{x}\right|^{2}+|x|^{2 \sigma}\left|u_{y}\right|^{2}\right)>N_{1}^{2}\right\}\right| \leq \epsilon\left|B_{1}\right|$. 
Then, for $\epsilon_{1}=C(\gamma) \epsilon$, the following inequalities holds,

(a) $\quad\left|B_{1} \cap\left\{\mathcal{M}\left(u_{x}^{2}+|x|^{2 \sigma} u_{y}^{2}\right)>N_{1}^{2}\right\}\right| \leq \epsilon_{1}\left(\left|B_{1} \cap\left\{\mathcal{M}\left(u_{x}^{2}+|x|^{2 \sigma} u_{y}^{2}\right)>1\right\}\right|\right.$

$$
\left.+\left|B_{1} \cap\left\{\mathcal{M}\left(|f|^{2}+|g|^{2}\right)>\delta^{2}\right\}\right|\right) \text {; }
$$

(b) $\left|B_{1} \cap\left\{\mathcal{M}\left(u_{x}^{2}+|x|^{2 \sigma} u_{y}^{2}\right)>N_{1}^{2} \lambda^{2}\right\}\right| \leq \epsilon_{1}\left(\left|B_{1} \cap\left\{\mathcal{M}\left(u_{x}^{2}+|x|^{2 \sigma} u_{y}^{2}\right)>\lambda^{2}\right\}\right|\right.$

$$
\left.+\left|B_{1} \cap\left\{\mathcal{M}\left(|f|^{2}+|g|^{2}\right)>\delta^{2} \lambda^{2}\right\}\right|\right) ;
$$

(c) $\quad\left|B_{1} \cap\left\{\mathcal{M}\left(u_{x}^{2}+|x|^{2 \sigma} u_{y}^{2}\right)>N_{1}^{2 k}\right\}\right| \leq \epsilon_{1}^{k}\left|B_{1} \cap\left\{\mathcal{M}\left(u_{x}^{2}+|x|^{2 \sigma} u_{y}^{2}\right)>1\right\}\right|$

$$
\begin{aligned}
& +\sum_{i=1}^{k} \epsilon_{1}^{i} \mid B_{1} \cap\left\{\mathcal{M}\left(|f|^{2}+|g|^{2}\right)\right. \\
> & \left.\delta^{2} N_{1}^{2(k-i)}\right\} \mid .
\end{aligned}
$$

Proof. The conclusion (a) comes from Lemma 2.4 and Corollary 4.7 by choosing

$$
C=\left\{X \in B_{1}: \mathcal{M}\left(\left|u_{x}\right|^{2}+|x|^{2 \sigma}\left|u_{y}\right|^{2}\right)>N_{1}^{2}\right\},
$$

and

$$
D=\left(\left\{\mathcal{M}\left(u_{x}^{2}+|x|^{2 \sigma} u_{y}^{2}\right)>N_{1}^{2}\right\} \cup\left\{\mathcal{M}\left(|f|^{2}+|g|^{2}\right)>\delta^{2}\right\}\right) \cap B_{1} .
$$

The conclusion (b) can be obtained by applying (a) to the weak solution $v(x, y)=$ $\frac{u(x, y)}{\lambda}$ of the equation

$$
v_{x x}+|x|^{2 \sigma} v_{y y}=\frac{f_{x}+|x|^{\sigma} g_{y}}{\lambda},
$$

(c) is an iteration of (b) by taking $\lambda=N_{1}^{i}, i=1,2 \ldots$

Now we go back to the proof of Theorem 4.1 .

Proof. By scaling, we need only to prove $r=1$.

When $p=2$, the theorem is easily seen by Lemma 4.2. the measure

Let $p>2$. We may assume that $\|f\|_{L^{p}\left(B_{5}\right)}$ and $\|g\|_{L^{p}\left(B_{5}\right)}$ are small, and also

$$
\left|\left\{X \in B_{1}, \mathcal{M}\left(\left|u_{x}\right|^{2}+|x|^{2 \sigma}\left|u_{y}\right|^{2}\right)>N_{1}^{2}\right\}\right| \leq \varepsilon\left|B_{1}\right|
$$

by multiplying the equation a small constant. Since $f$ and $g$ are in $L^{p}$ we have $|f|^{2}$ and $|g|^{2}$ are in $L^{\frac{p}{2}}$. By using Lemma 2.5 we have $\mathcal{M}\left(|f|^{2}+|g|^{2}\right) \in L^{\frac{p}{2}}$ with a small norm. We assume $\|f\|_{L^{p}\left(B_{5}\right)}+\|g\|_{L^{p}\left(B_{5}\right)} \leq \delta^{p}$, then

$$
\begin{aligned}
& \sum_{i=0}^{\infty} N_{1}^{i p}\left|\left\{\mathcal{M}\left(|f|^{2}+|g|^{2}\right)>\delta^{2} N_{1}^{2 i}\right\}\right| \\
& \leq \frac{C\left(\gamma, N_{1}, p\right)}{\delta^{p}}\left(\|f\|_{L^{p}}+\|g\|_{L^{p}}\right) \leq C .
\end{aligned}
$$


Thus

$$
\begin{aligned}
& \int_{B_{1}}\left|u_{x}\right|^{p}+\left.\left.|| x\right|^{\sigma} u_{y}\right|^{p} \\
& \leq \int_{B_{1}} \mathcal{M}\left(\left|u_{x}\right|^{2}+|x|^{2 \sigma}\left|u_{y}\right|^{2}\right)^{\frac{p}{2}} d x d y \\
& =p \int_{0}^{\infty} \lambda^{p-1}\left|B_{1} \cap\left\{\mathcal{M}\left(\left|u_{x}\right|^{2}+|x|^{2 \sigma}\left|u_{y}\right|^{2}\right) \geq \lambda^{2}\right\}\right| d \lambda \\
& \leq p\left(\left|B_{1}\right|+\sum_{k=0}^{\infty} \int_{N_{1}^{k}}^{N_{1}^{k+1}} \lambda^{p-1}\left|B_{1} \cap\left\{\mathcal{M}\left(\left|u_{x}\right|^{2}+|x|^{2 \sigma}\left|u_{y}\right|^{2}\right) \geq \lambda^{2}\right\}\right| d \lambda\right) \\
& \leq p\left|B_{1}\right|+p \sum_{k=0}^{\infty} N_{1}^{(k+1)(p-1)}\left|B_{1} \cap\left\{\mathcal{M}\left(\left|u_{x}\right|^{2}+|x|^{2 \sigma}\left|u_{y}\right|^{2}\right) \geq N_{1}^{2 k}\right\}\right| \\
& \leq p\left(1+\left|N_{1}^{p}\right|\right)\left|B_{1}\right|+N_{1}^{p} \sum_{k=1}^{\infty} N_{1}^{k p}\left(\epsilon_{1}^{k}\left|B_{1} \cap\left\{\mathcal{M}\left(u_{x}^{2}+|x|^{2 \sigma} u_{y}^{2}\right)>1\right\}\right|\right. \\
& \left.+\sum_{i=1}^{k} \epsilon_{1}^{i}\left|B_{1} \cap\left\{\mathcal{M}\left(|f|^{2}+|g|^{2}\right)>\delta^{2} N_{1}^{2(k-i)}\right\}\right|\right) \\
& \leq p\left(1+\left|N_{1}^{p}\right|\right)\left|B_{1}\right|+N_{1}^{p}\left(\sum_{k=1}^{\infty} N_{1}^{k p} \epsilon_{1}^{k}\left|B_{1} \cap\left\{\mathcal{M}\left(u_{x}^{2}+|x|^{2 \sigma} u_{y}^{2}\right)>1\right\}\right|\right. \\
& \left.+\sum_{i=1}^{\infty} \epsilon_{1}^{i} N_{1}^{i p} \sum_{k \geq i} N_{1}^{(k-i) p}\left|B_{1} \cap\left\{\mathcal{M}\left(|f|^{2}+|g|^{2}\right)>\delta^{2} N_{1}^{2(k-i)}\right\}\right|\right) \\
& \leq C \text {, }
\end{aligned}
$$

by taking $\epsilon_{1}$ small enough such that $N_{1}^{p} \epsilon_{1}<1$.

If $1<p<2$, then the statement follows from the standard duality argument, so the proof is finished.

Now we have all we needed to prove Theorem 1.1.

Proof. Let $\delta=d\left(\Omega^{\prime}, \partial \Omega\right)$.

For every point $Y \in \Omega^{\prime} \cap\{x=0\}$, consider the equation (1.1) in the ball $B(Y, \delta)$. Since the equation is translation invariant along $y$ axis, by Theorem 4.1 we have

$$
\begin{aligned}
& \int_{B\left(Y, \frac{\delta}{80 \gamma^{6}}\right)}\left(\left|u_{x}\right|^{p}+\left.\left.|| x\right|^{\sigma} u_{y}\right|^{p}\right) d x d y \\
& \leq C \int_{B\left(Y, \frac{\delta}{16 \gamma^{6}}\right)}\left(\left(\frac{\delta}{80 \gamma^{6}}\right)^{-p}|u|^{p}+|f|^{p}+|g|^{p}\right) d x d y .
\end{aligned}
$$


For every point $X_{0} \in \Omega^{\prime} \cap\left\{(x, y):|x|>\frac{\delta}{160 \gamma^{6}}\right\}$, we consider the equation (1.1) in the domain $B\left(X_{0}, \frac{\left|x_{0}\right|}{2}\right) \cap \Omega$ and let $v(x, y)$ be the scaling function of $u$ that is the same as in (4.12). Then $v(x, y)$ satisfies equation (4.13) and is uniformly elliptic in $B\left(\widetilde{X_{0}}, \frac{1}{2}\right) \cap \widetilde{\Omega}$, where $\widetilde{X_{0}}$ is the same as in (4.14) and $\widetilde{\Omega}=\left\{X:\left|x_{0}\right| X \in \Omega\right\}$. Applying the classical interior $W^{1, p}$ estimates for $v(x, y)$ and scaling back, we have

$$
\begin{aligned}
& \int_{B\left(X_{0}, \frac{\left|x_{0}\right|}{4}\right) \cap \Omega^{\prime}}\left(\left|u_{x}\right|^{p}+\left.\left.|| x\right|^{\sigma} u_{y}\right|^{p}\right) d x d y \\
& \leq C \int_{B\left(X_{0}, \frac{\left|x_{0}\right|}{2}\right) \cap \Omega}\left(|\delta|^{-p}|u|^{p}+|f|^{p}+|g|^{p}\right) d x d y .
\end{aligned}
$$

The estimate (1.2) follows by covering $\Omega^{\prime}$ with a finite number of balls $B\left(Y, \frac{\delta}{80 \gamma^{6}}\right)$ with $Y=(0, y)$ and $B\left(X, \frac{|x|}{4}\right)$ with $|x|>\frac{\delta}{160 \gamma^{6}}$.

Theorem 1.2 is easily derived by Theorem 1.1 and the odd extension method, so we omit the proof.

\section{References}

[1] S. S. BYUN and L. WANG, Elliptic equations with BMO coefficents in reifenberg domains, Comm. Pure Appl. Math. 57 (2004), 1283-1310.

[2] L. Capogna, D. Danielli and N. Garofalo, Subelliptic mollifiers and a basic pointwise estimate of Poincaré type, Math. Z. 226 (1997), 147-154.

[3] B. FRANCHI, Weighted Sobolev-Poincaré inequalities and pointwise estimates for a class of degenerate elliptic equations, Trans. Amer. Math. Soc. 327 (1991), 125-158.

[4] B. FRANCHI and E. LANCONELLI, Hölder regularity theorem for a class of linear nonuniformly elliptic operator with measurable coefficients, Ann. Scuola Norm. Sup. Pisa Cl. Sci. 10 (1983), 523-541.

[5] B. Franchi and R. SERAPIONI, Pointwise estimates for a class of strongly degenerate elliptic operators: a geometrical approach, Ann. Scuola Norm. Sup. Pisa Cl. Sci. 14 (1987), 527-568.

[6] N. Garofalo and D. M. NHIEU, Isoperimetric and the Sobolev inequalities for CarnotCarathéodory spaces and the existence of minimal surfaces, Comm. Pure Appl. Math. 49 (1996), 1081-1144.

[7] L. HöRmANDER, Hypoelliptic second order differential equations, Acta Math. 119 (1967), 147-171.

[8] D. JERISON, The Poincaré inequality for vector fields satisfying Hörmander's condition, Duke. Math. J. 53 (1986), 503-523.

[9] J. J. KoHn, Pseudo differential operators and Hypoellipticity, Proc. Symp. Pure Math. Amer. Math. Soc. 23 (1973), 61-69.

[10] E. LANCONELli and D. Morbidelli, On the Poincaré inequality for the vector fields, Ark. Mat. 38 (2000), 327-342.

[11] A. LOIUDICE, Sobolev inequalities with remainder terms for sublaplacians and other subelliptic operators, NoDEA Nonlinear Differential Equations Appl. 13 (2006), 119-136.

[12] L. P. RothSCHILD and E. M. STEIN, Hypoelliptic differential operator and nilpotent groups, Acta Math. 137 (1977), 247-320. 
[13] E. M. STEIN, “Singular Integrals and Differentiability Properties of Functions", Princeton, 1970.

[14] L. WANG, Hölder estimates for subelliptic opertors, J. Funct. Anal. 199 (2003), 228-242.

[15] L. WANG, A geometric approach to the Calderón-Zygmund estimates, Acta Math. Sin. (Engl. Ser.) 19 (2003), 381-396.

College of Science

Xi'an Jiaotong University

Shaanxi, 710049, China

songqzh@gmail.com

Business School

Jilin University

Jilin, 130012, China

luuying@163.com

College of Science

Xi'an Jiaotong University

Shaanxi, 710049, China

jzshen@mail.xjtu.edu.cn

Department of Mathematics

University of Iowa

IA 52246, USA

lwang@math.uiowa.edu 